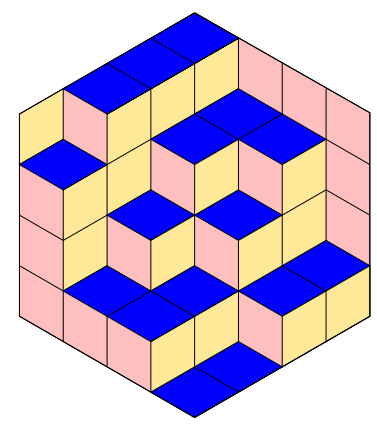

ALGEBRAIC COMBINATORICS

Gábor Somlai \& Mikhail Muzychuk

The Cayley isomorphism property for $\mathbb{Z}_{p}^{3} \times \mathbb{Z}_{q}$

Volume 4, issue 2 (2021), p. 289-299.

<http://alco.centre-mersenne.org/item/ALCO_2021__4_2_289_0>

(C) The journal and the authors, 2021.

Some rights reserved.

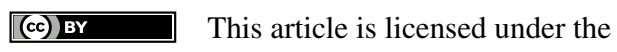

Creative Commons Attribution 4.0 International LiCEnSE.

http://creativecommons.org/licenses/by/4.0/

Access to articles published by the journal Algebraic Combinatorics on the website http://alco.centre-mersenne.org/ implies agreement with the Terms of Use (http://alco.centre-mersenne.org/legal/).

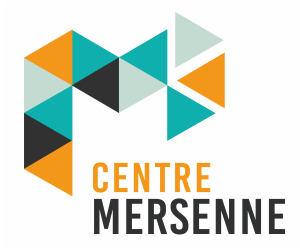

Algebraic Combinatorics is member of the Centre Mersenne for Open Scientific Publishing www.centre-mersenne.org 


\title{
The Cayley isomorphism property for $\mathbb{Z}_{p}^{3} \times \mathbb{Z}_{q}$
}

\author{
Gábor Somlai \& Mikhail Muzychuk
}

\begin{abstract}
For every pair of distinct primes $p, q$, where $q>2$ we prove that $\mathbb{Z}_{p}^{3} \times \mathbb{Z}_{q}$ is a CI-group with respect to binary relational structures.
\end{abstract}

\section{INTRODUCTION}

Let $H$ be a finite group and $S$ a subset of $G$. The Cayley digraph $\operatorname{Cay}(H, S)$ is defined by having the vertex set $H$ and $g$ is adjacent to $h$ if and only if $g h^{-1} \in S$. The set $S$ is called the connection set of the Cayley digraph Cay $(H, S)$. An undirected Cayley digraph will be referred to as a Cayley graph. Recall that a Cayley digraph $\operatorname{Cay}(H, S)$ is undirected if and only if $S=S^{-1}$, where $S^{-1}=\left\{s^{-1} \mid s \in S\right\}$. Every right multiplication via elements of $H$ is an automorphism of Cay $(H, S)$, so the automorphism group of every Cayley graph over $H$ contains a regular subgroup denoted by $\hat{H}$ isomorphic to $H$. Moreover, this property characterises the Cayley graphs of $H$.

By a binary Cayley structure (or a colored Cayley digraph) over $H$ we mean an ordered tuple (Cay $\left(H, S_{1}\right), \ldots$, Cay $\left(H, S_{r}\right)$ ) of Cayley digraphs, where $S_{i} \cap S_{j}=\varnothing$ if $i \neq j$, which we will abbreviate as $\operatorname{Cay}\left(H,\left(S_{1}, \ldots, S_{r}\right)\right)$. An isomorphism between two tuples Cay $\left(H,\left(S_{1}, \ldots, S_{r}\right)\right)$ and Cay $\left(H,\left(T_{1}, \ldots, T_{r}\right)\right)$ is a permutation $f \in \operatorname{Sym}(H)$ satisfying $\operatorname{Cay}\left(H, S_{i}\right)^{f}=\operatorname{Cay}\left(H, T_{i}\right), i=1, \ldots, r$. With this definition, the automorphism group of the binary Cayley structure Cay $\left(H,\left(S_{1}, \ldots, S_{r}\right)\right)$ coincides with $\bigcap_{i=1}^{r} \operatorname{Aut}\left(\operatorname{Cay}\left(H, S_{i}\right)\right)$.

It is clear that every automorphism $\mu$ of the group $H$ induces an isomorphism between Cay $\left(H,\left(S_{1}, \ldots, S_{r}\right)\right)$ and Cay $\left(H,\left(S_{1}^{\mu}, \ldots, S_{r}^{\mu}\right)\right)$. Such an isomorphism is called a Cayley isomorphism. A colored Cayley digraph $\operatorname{Cay}(G, \mathfrak{S})$, where $\mathfrak{S} \in\left(2^{H}\right)^{r}$ has the CI-property (or is a colored CI-digraph) if, for each $\mathfrak{T} \in \mathcal{P}\left(2^{H}\right)^{r}$ the colored Cayley digraph $\operatorname{Cay}(H, \mathfrak{T})$ is isomorphic to $\operatorname{Cay}(G, \mathfrak{S})$ if and only if they are Cayley isomorphic, i.e. there is an automorphism $\mu$ of $H$ such that $\mathfrak{S}^{\mu}=\mathfrak{T}$. In this case we say that $H$ has the CI-property for binary relational structures, or, it is a $\mathrm{CI}^{(2)}$-group. Note that the notion of $\mathrm{CI}^{(2)}$-groups was defined in a slightly different way in [12] but the two definitions are equivalent. Furthermore, a group $H$ is called a DCI-group if every Cayley digraph of $H$ is a CI-digraph and it is called a CI-group if every undirected Cayley digraph of $H$ is a CI-graph.

Manuscript received 29th July 2019, revised 10th August 2020, accepted 30th September 2020. KEYwORDS. Cayley graphs, CI property.

ACKNOWLEDGEMENTS. Mikhail Muzychuk was supported by the Israeli Ministry of Absorption. Gábor Somlai was supported by NKFIH SNN 132625. 
Investigation of the isomorphism problem of Cayley graphs started with Ádám's conjecture [1]. Using our terminology, it was conjectured that every cyclic group is a DCI-group. This conjecture was first disproved by Elspas and Turner [8] for directed Cayley graphs of $\mathbb{Z}_{8}$ and for undirected Cayley graphs of $\mathbb{Z}_{16}$.

Analyzing the spectrum of circulant graphs Elspas and Turner [8], and independently Djoković [5] proved that every cyclic group of order $p$ is a CI-group if $p$ is a prime. Also, a lot of research was devoted to the investigation of circulant graphs. One important result for our investigation is that $\mathbb{Z}_{p q}$ is a DCI-group for every pair of primes $p<q$. This result was first proved by Alspach and Parsons [2] and independently by Pöschel and Klin [13] using the theory of Schur rings, and also by Godsil [11]. Finally, Muzychuk [18, 19] proved that a cyclic group $\mathbb{Z}_{n}$ is a DCI-group if and only if $n=k$ or $n=2 k$, where $k$ is square-free. Furthermore, $\mathbb{Z}_{n}$ is a CI-group if and only if $n$ is as above or $n=8,9,18$.

It is easy to see that every subgroup of a (D)CI-group is also a (D)CI-group so it is natural to investigate $p$-groups which are the Sylow $p$-subgroups of a finite group. Babai and Frankl [4] proved that if $H$ is a $p$-group, which is a CI-group, then $H$ can only be an elementary abelian $p$-group, the quaternion group of order 8 or one of a few cyclic groups $\mathbb{Z}_{4}, \mathbb{Z}_{8}, \mathbb{Z}_{9}$ or $\mathbb{Z}_{27}$. The known results about cyclic groups show that $\mathbb{Z}_{27}$ is not a CI-group and $\mathbb{Z}_{9}, \mathbb{Z}_{8}$ are not DCI-groups. Babai and Frankl also asked whether every elementary abelian $p$-group is a (D)CI-group.

The cyclic group of order $p$, which is a CI-group, can also be considered as an elementary abelian $p$-group of rank 1 . Currently, the best general result is due to Feng and Kovács [10] who proved that $\mathbb{Z}_{p}^{5}$ is a CI-group for every prime $p$. The proof using elementary tools for $\mathbb{Z}_{p}^{4}$ is due to Morris [17]. It was shown by Somlai [22] that $\mathbb{Z}_{p}^{r}$ is not a DCI-group if $r \geqslant 2 p+3$.

Severe restrictions on the structure of DCI-groups were given by Li and Praeger and then a more precise list of candidates for DCI-groups was given by $\mathrm{Li}, \mathrm{Lu}$ and Pálfy [16]. A new family of CI-groups was found by Kovács and Muzychuk [14], that is, $\mathbb{Z}_{p}^{2} \times \mathbb{Z}_{q}$ is a DCI-group for every prime $p$ and $q$. One example of DCI-groups connected to the question treated in this paper is $\mathbb{Z}_{2}^{3} \times \mathbb{Z}_{p}$, see [6]. It was also conjectured in [14], that the direct product of DCI-groups of coprime order is a DCI-group ${ }^{(1)}$. Note that the conjecture is not true for CI-groups as it was shown recently by Dobson [7]. Dobson also proved that the product of relatively prime order elementary abelian DCI-groups is a DCI-group by posing a serious assumption on the prime divisors of the order of the group [6].

In this paper we prove the following result which supports this conjecture.

THEOREM 1.1. For every pair of primes $p$, $q$, where $q>2$ the group $\mathbb{Z}_{p}^{3} \times \mathbb{Z}_{q}$ is a DCI-group.

In fact we prove here a more general fact: the above group is a $\mathrm{CI}^{(2)}$-group. Our paper is organized as follows. In Section 2 we introduce the basic notation from Schur rings theory that is needed in this paper. In Section 3 we prove general results about Schur rings over abelian groups of special order. Finally, Section 4 contains the proof of Theorem 1.1.

\section{SCHUR RINGS}

This section is devoted to presenting a standard approach for dealing with the CIproblem via Schur rings so the results collected here are not new.

The result below is a direct consequence of Babai's lemma [3].

${ }^{(1)}$ The cited paper deals in fact with DCI-groups while it talks about CI-groups. 
Lemma 2.1. A colored Cayley graph $\mathrm{Cay}(H, \mathfrak{S}), \mathfrak{S} \in \mathcal{P}(H)^{r}$ has the CI-property if and only if any $H$-regular subgroup ${ }^{(2)}$ of the full automorphism group $\operatorname{Aut}(\operatorname{Cay}(H, \mathfrak{S}))$ is conjugate to $\hat{H}$ inside $\operatorname{Aut}(\operatorname{Cay}(H, \mathfrak{S}))$.

According to this result, in order to prove the CI-property for binary Cayley structures, it is sufficient to go through the whole set of automorphism groups of all colored Cayley graph over $H$. This could be done using the method of Schur rings. Let $G:=\operatorname{Aut}(\operatorname{Cay}(H, \mathfrak{S})), \mathfrak{S}=\left(S_{1}, \ldots, S_{r}\right)$ denote the full automorphism group of a colored digraph $\operatorname{Cay}(H, \mathfrak{S})$. Its intersection with $\operatorname{Aut}(H)$ will be denoted as $\operatorname{Aut}_{H}(\operatorname{Cay}(H, \mathfrak{S}))$. Let us order the orbits of $G_{e}$ in an arbitrary way, say $O_{1}, \ldots, O_{t}$. Since Aut $\left(\operatorname{Cay}\left(H,\left(S_{1}, \ldots, S_{r}\right)\right)\right)=\operatorname{Aut}\left(\operatorname{Cay}\left(H,\left(O_{1}, \ldots, O_{t}\right)\right)\right)$, we have to analyze only those colored Cayley graphs which correspond to overgroups $G \leqslant \operatorname{Sym}(H)$ of $\hat{H}$. It turns out that these colored Cayley graphs are closely related to Schur rings.

2.1. SCHUR RINGS OVER FINITE Groups. We start with the basic definitions [23]. Given a group $H$, we denote its group algebra over the rationals as $\mathbb{Q}[H]$. If $S \subseteq H$, then by $\underline{S}$ we denote the element $\sum_{s \in S} s \in \mathbb{Q}[H]$. Following [23] we call elements of this type simple quantities.

A subalgebra $\mathfrak{A}$ of the group ring $\mathbb{Q}[H]$ is called a $S$ chur ring, an $S$-ring for short, if it satisfies the following conditions.

(1) There exists a partition $\mathcal{T}=\left\{T_{0}, T_{1}, \ldots, T_{l}\right\}$ of $H$ such that $\mathfrak{A}$ is generated as a vector space by the elements of the following form: $\underline{T}=\sum_{t \in T} t$.

(2) $T_{0}=\{e\}$.

(3) For each $0 \leqslant i \leqslant l$ the subset $T_{i}^{(-1)}:=\left\{t^{-1} \mid t \in T_{i}\right\}^{(3)}$ belongs to $\mathcal{T}$.

The elements of the partition $\mathcal{T}$ are called basic sets of $\mathfrak{A}$ and $\underline{T}_{i}$ 's are called basic quantities. In what follows the notation $\operatorname{Bsets}(\mathfrak{A})$ will stand for $\mathcal{T}$ and any partition satisfying the above conditions will be referred to as a Schur partition. We say that a Schur ring is non-trivial if $H \backslash\{e\}$ is the union of at least two basic sets.

One of the most natural examples of Schur rings are the transitivity modules. Let $\hat{H} \leqslant \operatorname{Sym}(H)$ be the right regular representation of a finite group $H$ and $G \leqslant \operatorname{Sym}(H)$ its overgroup, i.e. $\hat{H} \leqslant G$. Then the orbits of the stabilizer $G_{e}$ are the basic sets of a Schur ring over $H$ [21]. Such a Schur ring will be called the transitivity module of $H$ induced by $G$ and denoted by $V\left(H, G_{e}\right)$. If $G=\hat{H} M$ for some $M \leqslant \operatorname{Aut}(H)$, then the Schur ring $V\left(H, G_{e}\right)$ is called cyclotomic. In this case, the basic sets of $V\left(H, G_{e}\right)$ coincide with the orbits of $M$.

Every Schur partition (equivalently every S-ring) $\mathcal{T}=\left\{T_{0}, \ldots, T_{d}\right\}$ gives rise to an association scheme $\operatorname{Cay}(H, \mathcal{T})$ whose basic graphs are the Cayley graphs $\operatorname{Cay}(H, T), T \in \mathcal{T}$. Two Schur partitions (Schur rings) $\mathfrak{A} \subseteq \mathbb{Q}[H], \mathfrak{B} \subseteq \mathbb{Q}[F]$ are called (combinatorially) isomorphic if the corresponding association schemes are isomorphic, i.e. there exists a bijection $f: H \rightarrow F$ which maps the basic Cayley graphs Cay $(H, T), T \in \mathcal{T}$ bijectively onto the set $\{\operatorname{Cay}(F, S)\}_{S \in \operatorname{Bsets}(\mathfrak{B})}$. The bijection $f$ is called a combinatorial isomorphism between $\mathfrak{A}$ and $\mathfrak{B}$. The isomorphism $f$ is called normalized if $f\left(e_{H}\right)=e_{F}$. If $f$ is a normalized isomorphism between $\mathfrak{A}$ and $\mathfrak{B}$, then $\operatorname{Bsets}(\mathfrak{A})^{f}=\operatorname{Bsets}(\mathfrak{B})$.

We denote by Iso $(\mathfrak{A}, \mathfrak{B})$ the set of all combinatorial isomorphisms between $\mathfrak{A}, \mathfrak{B}$ and by $\operatorname{Iso}_{e}(\mathfrak{A}, \mathfrak{B})$ its subset consisting of the normalized ones. It is easy to see that $\operatorname{Iso}(\mathfrak{A}, \mathfrak{B})=\hat{H} \operatorname{lso}_{e}(\mathfrak{A}, \mathfrak{B})=\operatorname{Iso}_{e}(\mathfrak{A}, \mathfrak{B}) \hat{F}$.

Note that Iso $(\mathfrak{A}, \mathfrak{B})$ is empty if and only if $\mathfrak{A}, \mathfrak{B}$ are not combinatorially isomorphic.

\footnotetext{
${ }^{(2)}$ An $H$-regular subgroup is any regular subgroup of the symmetric group isomorphic to $H$.

${ }^{(3)}$ The notation $T^{(-1)}$ is a particular case of a more general one $T^{(m)}$ introduced later.
} 
In what follows we write Iso $(\mathfrak{A}, *)$ for the union of Iso $(\mathfrak{A}, \mathfrak{B})$, where the second argument runs among all S-rings over the group $H$. As before, $\operatorname{Iso}(\mathfrak{A}, *)=\hat{H} \operatorname{Iso}_{e}(\mathfrak{A}, *)=\operatorname{Iso}_{e}(\mathfrak{A}, *) \hat{H}$.

Two S-rings $\mathfrak{A} \subseteq \mathbb{Q}[H]$ and $\mathfrak{B} \subseteq \mathbb{Q}[F]$ are Cayley isomorphic if there exists a group isomorphism $\varphi: H \rightarrow F$ such that $\varphi(\mathfrak{A})=\mathfrak{B}$. Note that Cayley isomorphic S-rings are always combinatorially isomorphic but not vice versa.

An S-ring $\mathfrak{A}$ is a $C I$-S-ring if for any S-ring $\mathfrak{A}^{\prime} \subseteq \mathbb{Q}[H]$ and arbitrary $f \in \operatorname{Iso}_{e}\left(\mathfrak{A}, \mathfrak{A}^{\prime}\right)$ there exists $\varphi \in \operatorname{Aut}(H)$ such that $f(S)=\varphi(S)$ for all $S \in \operatorname{Bsets}(\mathfrak{A})$. It follows directly from the definition that an S-ring $\mathfrak{A}$ is a CI-S-ring if and only if Iso( $\mathfrak{A}, *)=$ $\operatorname{Aut}(\mathfrak{A}) \operatorname{Aut}(H)$, or, equivalently, $\operatorname{Iso}_{e}(\mathfrak{A}, *)=\operatorname{Aut}(\mathfrak{A})_{e} \operatorname{Aut}(H)$. Note that the definition of a CI-S-ring given in [12] was based on the first equality.

As an application of Babai's lemma [3] we have the following statement [12].

Proposition 2.2. Let $\Gamma:=\operatorname{Cay}(H, \Sigma)$ be a colored Cayley graph over $H$ and $G:=$ Aut $(\Gamma)$. The following are equivalent

(1) $\Gamma$ has the CI-property;

(2) any $H$-regular subgroup of $G$ is conjugate to $\hat{H}$ in $G$;

(3) the transitivity module $V\left(H, \operatorname{Aut}(\Gamma)_{e}\right)$ is a $C I$-S-ring.

This implies the following result.

THEOREM 2.3. A group $H$ has a CI-property for binary relational structures $\left(C I^{(2)}\right.$ group, for short) if and only if every transitivity module over $H$ is a CI-S-ring.

Thus one has to check all transitivity modules over the group $H$. To reduce the number of checks we use the following partial order on the set $\operatorname{Sup}(\hat{H})$ consisting of all overgroups of $\hat{H}$.

Given two overgroups $X, Y \in \operatorname{Sup}(\hat{H})$, we write $X \preceq_{\hat{H}} Y$ if any $H$-regular subgroup of $Y$ may be conjugated into $X$ by an element of $Y$, i.e.

$$
\forall_{g \in \operatorname{Sym}(H)}: \hat{H}^{g} \leqslant Y \Rightarrow \exists y \in Y:\left(\hat{H}^{g}\right)^{y} \leqslant X .
$$

One can easily check that $\preceq_{\hat{H}}$ is a partial order on the set of all overgroups of $\hat{H}$. Note that any two $H$-regular subgroups of $X \in \operatorname{Sup}(\hat{H})$ are conjugate inside $X$ if and only if $\hat{H} \preceq \hat{H} X$.

The statement below allows us to consider transitivity modules of $\preceq_{\hat{H}^{-m i n i m a l}}$ groups only.

Proposition 2.4. Let $G_{1} \leqslant G_{2}$ be two overgroups of $\hat{H}$ and $\mathfrak{A}_{i}:=V\left(H,\left(G_{i}\right)_{e}\right)$ their transitivity modules. Then $\mathfrak{A}_{1} \supseteq \mathfrak{A}_{2}$. If $G_{1} \preceq_{\hat{H}}$ Aut $\left(\mathfrak{A}_{2}\right)$ and $\mathfrak{A}_{1}$ is CI, then $\mathfrak{A}_{2}$ is also a $C I$-S-ring.

Proof. First we note that the inclusion $\mathfrak{A}_{1} \supseteq \mathfrak{A}_{2}$ is obvious.

To show the CI-property of $\mathfrak{A}_{2}$ we have to verify that Iso $\left(\mathfrak{A}_{2}, *\right) \subseteq \operatorname{Aut}\left(\mathfrak{A}_{2}\right) \operatorname{Aut}(H)$ (the converse inclusion is obvious). Pick an arbitrary $f \in \operatorname{Iso}\left(\mathfrak{A}_{2}, *\right)$. Then $\mathfrak{A}_{2}^{f}=\mathfrak{B}$ for some S-ring $\mathfrak{B}$ over $H$. Then $\hat{H} \leqslant \operatorname{Aut}(\mathfrak{B})=\operatorname{Aut}\left(\mathfrak{A}_{2}\right)^{f}$ implying $\hat{H}^{f^{-1}} \leqslant \operatorname{Aut}\left(\mathfrak{A}_{2}\right)$. It follows from the assumption that there exists $g \in \operatorname{Aut}\left(\mathfrak{A}_{2}\right)$ such that $\left(\hat{H}^{f^{-1}}\right)^{g} \leqslant G_{1}$. Combining this with $G_{1} \leqslant \operatorname{Aut}\left(\mathfrak{A}_{1}\right)$ we conclude that $\hat{H}^{f^{-1} g} \leqslant \operatorname{Aut}\left(\mathfrak{A}_{1}\right)$. Since $\mathfrak{A}_{1}$ is a CI-S-ring, there exists $g_{1} \in \operatorname{Aut}\left(\mathfrak{A}_{1}\right)$ such that $\hat{H}^{g_{1}}=\hat{H}^{f^{-1} g}$. This implies $f^{-1} g g_{1}^{-1} \in \hat{H} \operatorname{Aut}(H)$, or, equivalently, $g_{1} g^{-1} f \in \hat{H} \operatorname{Aut}(H)$. It follows from $\mathfrak{A}_{1} \supseteq \mathfrak{A}_{2}$ that Aut $\left(\mathfrak{A}_{1}\right) \subseteq \operatorname{Aut}\left(\mathfrak{A}_{2}\right)$. Therefore $g_{1} g^{-1} \in \operatorname{Aut}\left(\mathfrak{A}_{2}\right)$, and, consequently, $f \in \operatorname{Aut}\left(\mathfrak{A}_{2}\right) \operatorname{Aut}(H)$, as required. 
Sylow's theorem shows that if $H$ is a $p$-group, then any $\preceq_{\hat{H}}$-minimal overgroup of $\hat{H}$ is a $p$-group. In this case we are left to investigate transitivity modules whose basic sets have a $p$-power cardinality. These Schur rings are called $p$-Schur rings.

2.2. Structural properties of Schur Rings. As before, $H$ is a finite group and $\mathbb{Q}[H]$ is its group algebra. For an element of the group algebra $U=\sum_{g \in H} a_{g} g$ let $U^{(m)}=\sum_{g \in H} a_{g} g^{m}$. We extend this notation to an arbitrary subset $T$ of $H$ by $T^{(m)}=\left\{t^{m} \mid t \in T\right\}$.

The two lemmas below are taken from [23].

Lemma 2.5. Let $\mathfrak{A}$ be an $S$-ring over an abelian group $H$. If $\operatorname{gcd}(m,|H|)=1$, then $T^{(m)} \in \mathfrak{A}$ for every $T \in \mathfrak{A}$.

A similar statement holds if $m$ divides $|H|$.

Lemma 2.6. Let $\underline{T}$ be a simple quantity and $m$ a prime divisor of $|G|$ and let $\underline{T}^{m}=$ $\sum_{h \in H} a_{h} h$. Then for any integer $i$ the simple quantity $\sum_{h \in H \mid a_{h} \equiv i(\bmod m)} h$ belongs to $\mathfrak{A}$.

A subgroup $L \leqslant H$ is called an $\mathfrak{A}$-subgroup if $\underline{L} \in \mathfrak{A}$. We say that $\mathfrak{A}$ is primitive if the only $\mathfrak{A}$-subgroups are $\{e\}$ and $H$. A Schur ring $\mathfrak{A}$ is called imprimitive if $\underline{L} \in \mathfrak{A}$ for some non-trivial and proper subgroup $L \leqslant H$.

If $T$ is an $\mathfrak{A}$-set, then we may define its radical $\operatorname{Rad}(T)=\{g \in T \mid T g=g T=T\}$. It is well known that the radical of an $\mathfrak{A}$-set $T$ is an $\mathfrak{A}$-subgroup [23].

It is a simple observation that a trivial S-ring is always primitive. The converse is not true (e.g. [23, Theorem 25.7]). The result below proved by Wielandt ([23, Theorem 25.4]) provides a sufficient condition for the converse implication.

TheOREm 2.7. A primitive S-ring over an abelian group $H$ of a composite order is trivial if $H$ has a cyclic Sylow subgroup.

For an $\mathfrak{A}$-subgroup $U$ one can define $\mathfrak{A}_{U}$ as the restriction of $\mathfrak{A}$ to $U$ spanned by the basic sets of $\mathfrak{A}$ contained in $U$. For a pair of $\mathfrak{A}$-subgroups $L \unlhd U$ we define $\mathfrak{A}_{U / L}$ as a subring of $\mathbb{Q}[U / L]$ spanned by $\left\{\underline{X}^{\pi} \mid X \subset U, X \in \operatorname{Bsets}(\mathfrak{A})\right\}$, where $\pi$ denotes the canonical epimorphism from $U$ to $U / L[9]$.

We say that the Schur ring $\mathfrak{A}$ is a generalized wreath product if there exists $\mathfrak{A}$ subgroups $L \leqslant U$ such that $L$ is a normal subgroup in $H$ and every basic set outside of $U$ is the union of $L$-cosets. Such a wreath product is called trivial if $L=\{e\}$ or $U=H$. In the case of $L=U$ we obtain the usual wreath product of Schur rings.

Let $K$ and $L$ be two $\mathfrak{A}$-subgroups. We say that $\mathfrak{A}$ is the star product of $\mathfrak{A}_{K}$ and $\mathfrak{A}_{L}$ (or $\mathcal{A}$ admits a star decomposition) if the following conditions hold:

(1) $K \cap L \unlhd L$

(2) each basic set $T$ of $\mathfrak{A}$ with $T \subseteq(L \backslash K)$ is the union of $K \cap L$-cosets

(3) for each basic set $T \subseteq H \backslash(K \cup L)$ there exists $R, S \in \operatorname{Bsets}(\mathfrak{A})$, where $R \subseteq K$, $S \subseteq L$ such that $T=R S$.

Note that in order to verify (3) it is enough to find $\mathfrak{A}$-sets $R^{\prime}$ and $S^{\prime}$ with $T=R^{\prime} S^{\prime}$.

In this case we write $\mathfrak{A}=\mathfrak{A}_{K} \star \mathfrak{A}_{L}$. A star-decomposition is called trivial if $K=$ $\{e\}$ or $H$. In the case of $L=H$ a star decomposition coincides with the wreath product of $\mathfrak{A}_{K}$ and $\mathfrak{A} / K$.

The theorems below provide us sufficient conditions for these products to have the CI-property. Although the first statement was originally proved for elementary abelian groups only [12], the proof works for a more general class of groups, namely: the abelian groups with elementary abelian Sylow subgroups. In what follows we refer to these groups as $\mathcal{E}$-groups. 
Theorem 2.8 ([14, Theorem 3.2]). Let $H$ be an $\mathcal{E}$-group and let $G \leqslant \operatorname{Sym}(H)$ be an overgroup of $\hat{H}$. Assume that the transitivity module $\mathfrak{A}:=V\left(H, G_{e}\right)$ admits a nontrivial star-decomposition $\mathfrak{A}_{K} \star \mathfrak{A}_{L}$. If $\mathfrak{A}_{K}$ and $\mathfrak{A}_{L / K \cap L}$ are CI-S-rings, then $\mathfrak{A}$ is a $C I$-S-ring.

Note that the above theorem implies that if $\mathfrak{A}$ admits a usual wreath product decomposition, then $\mathfrak{A}$ is a CI-S-ring. In the case of a generalized wreath product we have the following result.

TheOREM 2.9 ([15]). Let $H$ be an $\mathcal{E}$-group and let $G \leqslant S y m(H)$ be an overgroup of $\hat{H}$. Assume that $\mathfrak{A}:=V\left(H, G_{e}\right)$ is a non-trivial generalized wreath product with respect to $\mathfrak{A}$-subgroups $\{e\} \neq L \leqslant U \neq H$. Assume that $\mathfrak{A}_{U}$ and $\mathfrak{A}_{H / L}$ are CI-S-rings and $\operatorname{Aut}_{U / L}\left(\mathfrak{A}_{U / L}\right)=\operatorname{Aut}_{U}\left(\mathfrak{A}_{U}\right)^{U / L} \operatorname{Aut}_{H / L}\left(\mathfrak{A}_{H / L}\right)^{U / L}$. Then $\mathfrak{A}$ is a CI-S-ring.

\section{SCHUR RINGS OVER ABELIAN GROUP OF NON-POWERFUL ORDER}

Recall that a number $n$ is call powerful if $p^{2}$ divides $n$ for every prime divisor $p$ of $n$. In this section and in what follows we assume that $H$ is an abelian group of a non-powerful order, i.e. there exists a prime divisor $q$ of $|H|$ such that $|H|=n q$ where $n$ is coprime to $q$. In what follows we call such $q$ a simple prime divisor of $|H|$. We assume that $q>2$.

Let $P$ and $Q$ denote the unique subgroups of $H$ of orders $n$ and $q$, respectively and let $Q^{\#}=Q \backslash\{1\}$. Let $\ell$ be the exponent of $P$. The group $\mathbb{Z}_{\ell q}^{*} \cong \mathbb{Z}_{\ell}^{*} \times \mathbb{Z}_{q}^{*}$ acts on $H$ via raising to the power as $h \mapsto h^{t}$, where $t \in \mathbb{Z}_{\ell q}^{*}$. Denote $M_{q}:=\left\{t \in \mathbb{Z}_{\ell q}^{*} \mid t \equiv 1\right.$ $(\bmod \ell)\}$. Clearly $M_{q} \cong \mathbb{Z}_{q}^{*}$.

Every element $h \in H$ has a unique decomposition into the product $h=h_{q^{\prime}} h_{q}$ where $h_{q^{\prime}} \in P$ and $h_{q} \in Q$. Notice that two elements $h, f \in H$ belong to the same $Q$-coset if and only if $h_{q^{\prime}}=f_{q^{\prime}}$. Let $q^{*} \in \mathbb{Z}_{\ell q}^{*}$ be an element satisfying $q^{*} q \equiv 1(\bmod \ell)$ and $q^{*} \equiv 1(\bmod q)$. Then $h_{q^{\prime}}=h^{q q^{*}}$.

Given a subset $T \subseteq H$. We write $T_{q^{\prime}}$ for the set $\left\{h_{q^{\prime}} \mid h \in T\right\}$. Notice that $T_{q^{\prime}}$ is always contained in $P$. We always have the decomposition $T=\bigcup_{s \in T_{q^{\prime}}} s R_{s}$ where $R_{s}:=s^{-1} T \cap Q$.

In what follows $\mathfrak{A}$ stands for a non-trivial S-ring over $H$. Let $P_{1}$ be the maximal $\mathfrak{A}$-subgroup contained in $P$ while $Q_{1}$ is the minimal $\mathfrak{A}$-subgroup which contains $Q$.

The statement below describes the structure of $M_{q}$-invariant basic sets.

Proposition 3.1. Let $T$ be a basic set of $\mathfrak{A}$ which is $M_{q}$-invariant. Denote $S:=T_{q^{\prime}}$. There exists a partition ${ }^{(4)} S=S_{1} \cup S_{-1} \cup S_{0}$ such that $T=S_{1} \cup S_{-1} Q^{\#} \cup S_{0} Q$ and $S_{1}, S_{-1}$ are $\mathfrak{A}$-subsets (not necessarily basic). In addition the sets $S_{1}, S_{-1}$ and $S_{0}$ satisfy the following conditions

(1) If $S_{1} \neq \varnothing$, then $S_{-1}=S_{0}=\varnothing$ and $T \subseteq P_{1}$;

(2) If $S_{1}=\varnothing$ and $S_{-1} \neq \varnothing$, then $T=S_{-1}\left(Q_{1} \backslash P_{1}\right)$;

(3) If $S_{1}=S_{-1}=\varnothing$, then $Q_{1} T=T$.

Proof. Write $T=\bigcup_{s \in S} s R_{s}$ where $R_{s}:=s^{-1} T \cap Q$. Since $T$ is $M_{q}$-invariant, the sets $R_{s}$ are $\mathbb{Z}_{q}^{*}$-invariant. Therefore $R_{s} \in\left\{\{1\}, Q^{\#}, Q\right\}$. Now the sets

$$
S_{1}:=\left\{s \mid R_{s}=\{1\}\right\}, S_{-1}:=\left\{s \mid R_{s}=Q^{\#}\right\}, S_{0}:=\left\{s \mid R_{s}=Q\right\}
$$

produce the required partition. Raising the simple quantity $\underline{T}=\underline{S_{1}}+\underline{S_{-1}} \cdot \underline{Q^{\#}}+\underline{S_{0}} \cdot \underline{Q}$ to the $q$-th power modulo $q$ we obtain

$$
\underline{T}^{q} \equiv\left(\underline{S}_{1}\right)^{q}-\left(\underline{S_{-1}}\right)^{q} \equiv\left(\underline{S_{1}^{(q)}}\right)-\left(\underline{S_{-1}^{(q)}}\right) \quad(\bmod q) .
$$

${ }^{(4)}$ Notice that some of its parts may be empty. 
Now Lemma 2.6 applied to $\underline{T}^{q}$ with $m=q$ and $i= \pm 1(-1 \neq 1$, because $q>2)$ implies that $S_{1}^{(q)}, S_{-1}^{(q)}$ are $\mathfrak{A}$-subsets. Applying $q^{*}$ we conclude that $S_{1}$ and $S_{-1}$ are A-subsets too.

If $S_{1} \neq \varnothing$, then $S_{1}=T$ because $T$ is basic and $S_{1}$ is a nonempty $\mathfrak{A}$-subset contained in $T$. Hence $S_{-1}=S_{0}=\varnothing$.

Assume now that $S_{1}=\varnothing$ and $S_{-1} \neq \varnothing$. Since $Q_{1} \backslash P_{1}=Q_{1} \backslash\left(Q_{1} \cap P_{1}\right)$ is an $\mathfrak{A}$-subset which contains $Q^{\#}$, we conclude that $S_{-1}\left(Q_{1} \backslash P_{1}\right)$ is an $\mathfrak{A}$-subset which intersects $T$ non-trivially (the part $S_{-1} Q^{\#}$ is in common). Therefore $S_{-1}\left(Q_{1} \backslash P_{1}\right) \supseteq T$.

The union $S_{-1} \cup T=\left(S_{-1} \cup S_{0}\right) Q$ is an $\mathfrak{A}$-subset the radical of which contains $Q$. Therefore, by the minimality of $Q_{1}$, we have $Q_{1} \leqslant \operatorname{Rad}\left(S_{-1} \cup T\right)$. This implies $Q_{1} S_{-1} \cup Q_{1} T=S_{-1} \cup T$ so $S_{-1} Q_{1} \subseteq S_{-1} \cup T$. Thus $T \subseteq S_{-1}\left(Q_{1} \backslash P_{1}\right) \subseteq S_{-1} \cup T$. If $S_{-1}\left(Q_{1} \backslash P_{1}\right) \cap S_{-1} \neq \varnothing$, then $s t=s^{\prime}$ for some $s, s^{\prime} \in S_{-1}$ and $t \in Q_{1} \backslash P_{1}$. But in this case we would obtain $t=s^{\prime} s^{-1} \subseteq S_{-1} S_{-1}^{(-1)} \subseteq P_{1}$, a contradiction. Hence $S_{-1}\left(Q_{1} \backslash P_{1}\right) \cap S_{-1}=\varnothing$ implying that $T=S_{-1}\left(Q_{1} \backslash P_{1}\right)$.

If $S_{1}=S_{-1}=\varnothing$. then $T=S_{0} Q$ so $\operatorname{Rad}(T)$ contains $Q$ By the minimality of $Q_{1}$ we have $Q_{1} \leqslant \operatorname{Rad}(T)$ so $Q_{1} T=T$.

COROLlary 3.2. $\mathfrak{A}$ is a generalized wreath product with respect to $Q_{1}$ and $P_{1} Q_{1}$.

Proof. There is nothing to prove if $Q_{1} P_{1}=H$. So, in what follows we assume that $Q_{1} P_{1} \neq H$.

We have to show that $Q_{1} T=T$ holds for each $\mathfrak{A}$-basic set $T$ outside of $P_{1} Q_{1}$. Let $T$ be such a basic set, that is, $T \cap P_{1} Q_{1}=\varnothing$.

If $T$ contains a $q^{\prime}$-element, then $T$ is $M_{q}$-invariant, and therefore, $T$ fits one of the cases described in Proposition 3.1. The cases (a) and (b) contradict $T \cap P_{1} Q_{1}=\varnothing$, since in both of them $T \subseteq P_{1} Q_{1}$. Therefore the case 3 of Proposition 3.1 occurs and $T Q_{1}=T$, as required.

It remains to show that every basic $\mathfrak{A}$-set disjoint with $P_{1} Q_{1}$ contains $q^{\prime}$-elements. Assume that there exists one, say $T$, which does not contain a $q^{\prime}$-element. Denote $R:=T_{q^{\prime}}$. Then $T$ can uniquely be written as $T=\cup_{h \in R} h Q_{h}$, where $Q^{\#} \supseteq Q_{h} \neq \varnothing$. Then by Lemma 2.6 $T^{(q)}=R^{(q)}$ is an $\mathfrak{A}$-set, implying that $R^{(q)} \subseteq P_{1}$ and $R \subseteq P_{1}$. Again we have $T \subseteq R Q \subseteq P_{1} Q_{1}$, contrary to the choice of $T$.

3.1. The structure of The SeCtion $\mathfrak{A}_{P_{1} Q_{1}}$. In what follows we abbreviate $H_{1}:=$ $P_{1} Q_{1}$ and $\mathfrak{A}_{1}:=\mathfrak{A}_{H_{1}}$. We start with the following simple statement.

Proposition 3.3. $P_{1}$ is an $\mathfrak{A}_{\mathbf{1}}$-maximal subgroup.

Proof. Let $\tilde{P}_{1}$ denote a proper $\mathfrak{A}_{1}$-maximal subgroup which contains $P_{1}$. If $q$ divides $\tilde{P}_{1}$, then $Q_{1}$ is contained in $\tilde{P}_{1}$ implying $P_{1} Q_{1} \leqslant \tilde{P}_{1}=H_{1}$, a contradiction. Hence $\tilde{P}_{1}$ is a $p$-group, which is an $\mathfrak{A}_{1}$-subgroup. Therefore, $\tilde{P}_{1}=P_{1}$.

Proposition 3.4. If $\left|H_{1} / P_{1}\right| \neq q$, then $\mathfrak{A}_{1} / P_{1}$ has rank two and $\mathfrak{A}_{1}=\left(\mathfrak{A}_{1}\right)_{P_{1}} \star\left(\mathfrak{A}_{1}\right)_{Q_{1}}$.

Proof. $P_{1}$ is an $\mathfrak{A}_{1}$-maximal subgroup, by Proposition 3.3. Thus the quotient S-ring is primitive. The Sylow $q$-subgroup of $H_{1} / P_{1}$ is cyclic. Therefore by Wielandt's Theorem 2.7 either the quotient S-ring has rank two or $H_{1} / P_{1}$ is of prime order. In the latter case, $\left|H_{1} / P_{1}\right|=q$, which contradicts our assumptions.

The quotient S-ring $\mathfrak{A}_{1} / P_{1}$ has rank two iff $T P_{1}=H_{1} \backslash P_{1}$ holds for each basic set $T \in \operatorname{Bsets}\left(\mathfrak{A}_{1}\right)$ outside of $P_{1}$.

It follows from $\left|H_{1} / P_{1}\right| \neq q$ that $P_{1} \neq\left(H_{1}\right)_{q^{\prime}}$. Pick an arbitrary $T \in \operatorname{Bsets}\left(\mathfrak{A}_{1}\right)$ with $T \cap P_{1}=\varnothing$. Then $T P_{1}=H_{1} \backslash P_{1} \supseteq\left(H_{1}\right)_{q^{\prime}} \backslash P_{1}$ implying $T \cap\left(H_{1}\right)_{q^{\prime}} \neq \varnothing$. Thus $T$ contains $q^{\prime}$-elements, and, therefore, is $M_{q}$-invariant and Proposition 3.1 is applicable. 
The first case of the Proposition is not possible because $T \cap P_{1}=\varnothing$.

In the second case we obtain that $T$ is the product of two $\mathfrak{A}_{1}$-sets $S_{-1} \subset P_{1}$ and $Q_{1} \backslash P_{1} \subset Q_{1}$ so $T$ fits the definition of star decomposition.

Finally, if $Q_{1} T=T$, then $T$ is the union of $Q_{1}$-cosets. Since $P_{1} Q_{1}=H_{1}$ we have that $P_{1}$ intersects every $Q_{1}$-coset. Hence $T \cap P_{1} \neq \varnothing$, contradicting the choice of $T$.

Thus, we have proven that any basic set $T$ of $\mathfrak{A}_{1}$ disjoint to $P_{1}$ has the form $S\left(Q_{1} \backslash P_{1}\right)$ where $S \subseteq P_{1}$ is an $\mathfrak{A}_{1}$-subset so is a union of $P_{1} \cap Q_{1}$-cosets. This immediately implies that $Q_{1} \backslash P_{1}$ is a basic set of $\mathfrak{A}_{1}$ and $\mathfrak{A}_{1}=\left(\mathfrak{A}_{1}\right)_{P_{1}} \star\left(\mathfrak{A}_{1}\right)_{Q_{1}}$.

Note that it follows from the Corollary 3.2 that if $H_{1}=Q_{1}$, then $\mathfrak{A}$ is a wreath product with respect to $P_{1}$.

$P_{1}$ is a maximal $\mathfrak{A}_{1}$-subgroup by Proposition 3.3 , and the order of $H_{1} / P_{1}$ is divisible by $q$ but not divisible by $q^{2}$. Thus by Theorem 2.7 if $\mathfrak{A}_{1} / P_{1}$ is non-trivial, then $\mathfrak{A}_{1} / P_{1}$ is a non-trivial S-ring over a cyclic group of order $q$. In particular, $\left[H_{1}: P_{1}\right]=q$. Although the structure of S-rings over $C_{q}$ is known [20] we do not need it, because for our purposes we need to settle the case when $\mathfrak{A}_{1} / P_{1}$ coincides with full group algebra.

From now on we denote the cyclic group of order $m$ by $C_{m}$ in order to make the notation more readable.

Proposition 3.5. If $\mathfrak{A}_{1} / P_{1} \cong \mathbb{Z}\left[C_{q}\right]$, then $\mathfrak{A}_{1}=\left(\mathfrak{A}_{1}\right)_{P_{1}} \star\left(\mathfrak{A}_{1}\right)_{Q_{1}}$.

Proof. It follows from the assumption that cosets $h P_{1}, h \in Q^{\#}$ are $\mathfrak{A}_{1}$-subsets. Therefore $h P_{1}$ is partitioned into a disjoint union of basic sets yielding a partition $\Sigma_{h}$ of $P_{1}$ :

$$
S \in \Sigma_{h} \Longleftrightarrow h S \in \operatorname{Bsets}\left(\mathfrak{A}_{1}\right) .
$$

Since $M_{q}$ permutes basic sets and acts transitively on $Q^{\#}$, the partitions $\Sigma_{h}$ does not depend on the choice of $h \in Q^{\#}$ by Lemma 2.5. So, in what follows we write just $\Sigma$ without an index.

Pick a basic set $T$ outside of $P_{1}$. Then $T=h S$ for some $h \in Q^{\#}$ and $S \in \Sigma$. Now it follows from $\underline{T}^{q} \equiv \underline{S}^{(q)}(\bmod q)$ that $S^{(q)}$ is an $\mathfrak{A}_{1}$-subset contained in $P_{1}$. Applying $q^{*}$ to $S^{(q)}$ we conclude that $S$ is an $\mathfrak{A}_{1}$-subset.

Since $\left\langle\underline{T} \mid T \in \operatorname{Bsets}\left(\mathfrak{A}_{1}\right) \wedge T \subseteq h P_{1}\right\rangle$ is an $\left(\mathfrak{A}_{1}\right)_{P_{1}}$-invariant subspace, the linear span $\underline{\Sigma}:=\langle\underline{S}\rangle_{S \in \Sigma}$ is an ideal of $\left(\mathfrak{A}_{1}\right)_{P_{1}}$. Let $S_{e} \in \Sigma$ be a class containing $e$.

We claim that $S_{e}$ is an $\mathfrak{A}_{e}$-subgroup and every class of $\Sigma$ is a union of $S_{e}$-cosets. This will imply our claim.

Pick a basic set $T$ of $\left(\mathfrak{A}_{1}\right)_{P_{1}}$ contained in $S_{e}$. Then $e$ appears in the product $\underline{T}^{(-1)} \underline{S_{e}}$ with coefficient $|T|$. Therefore $\underline{S}_{e}$ appears $|T|$ times in this product. This implies $\underline{T}^{(-1)} \underline{S_{e}}=|T| \underline{S_{e}}$ and, consequently, $T^{(-1)} S_{e}=S_{e}$. Since this equality holds for any basic set $T$ contained in $S_{e}$, we conclude that $S_{e}^{(-1)} S_{e}=S_{e}$, hereby proving that $S_{e}$ is a subgroup of $P_{1}$.

Pick now an arbitrary $S \in \Sigma$. Then $\underline{S}^{(-1)} \underline{S} \in \underline{\Sigma}$. The identity $e$ appear in the product $|S|$ times. Therefore $S_{e}$ appears in the product $\underline{S}^{(-1)} \underline{S}$ with coefficient $|S|$. Therefore $S$ is a union of $S_{e}$-cosets.

It is easy to see that $S_{e} h$ generates an $\mathfrak{A}_{1}$-subgroup, whose order is divisible by $q$ so it contains $Q_{1}$. On the other hand $S_{e} h$ is a basic set intersecting $Q$ non-trivially so it is contained in $Q_{1}$. Thus $S_{e}=Q_{1} \cap P_{1}$, which gives that $\mathfrak{A}_{1}$ admits a star decomposition.

\section{Proof of the main Result}

In this section we show that every transitivity module over the group $H \cong C_{p}^{3} \times C_{q}, p \neq$ $q$ are primes, is a CI-S-ring. Since $q$ is a simple prime divisor of $|H|$, the structural 
results from the previous section are applicable. We also keep the notation $P_{1}$ and $Q_{1}$ defined in Section 3.

For the rest of the section $\mathfrak{A}=V\left(H, G_{e}\right)$ is a transitivity module of an $\preceq_{\hat{H}^{-m i n i m a l}}$ subgroup $G$.

In this section we prove the following.

THEOREM 4.1. $\mathfrak{A}$ is a CI-S-ring.

Combining this result with Theorem 2.3 we obtain the main result of the paper.

4.1. Proof of Theorem 4.1 in the CASE of $P_{1} Q_{1} \neq H$. If $P_{1} Q_{1} \neq H$, then by Corollary 3.2 the S-ring $\mathfrak{A}$ is a non-trivial generalized wreath product of $\mathfrak{A}_{P_{1} Q_{1}}$ and $\mathfrak{A}_{H / Q_{1}}$. Therefore, the results of [15] are applicable.

Since $\bar{H}:=H / Q_{1}$ is an elementary abelian $p$-group, we may assume that the basic sets of $\overline{\mathfrak{A}}:=\mathfrak{A} / Q_{1}$ are of $p$-power length. Such a Schur ring is called a $p$-S-ring and so $\overline{\mathfrak{A}}$ is a transitivity module of the quotient group $\bar{G}:=G^{H / Q_{1}}$. Since $G$ is $\preceq_{H^{-} \text {-minimal, }}$ the group $\bar{G}$ is a $\preceq \bar{H}$-minimal.

If $\left|P_{1} Q_{1} / Q_{1}\right| \leqslant p$, then $\mathfrak{A}_{P_{1} Q_{1} / Q_{1}}$ is the full group ring and we are done by Proposition 4.1 of [15]. Thus we may assume that $\left|P_{1} Q_{1} / Q_{1}\right|=p^{a}$ with $a \geqslant 2$. Since $q$ divides $\left|P_{1} Q_{1}\right|$ and $P_{1} Q_{1} \neq H$, we conclude that $\left|P_{1}\right|=p^{2},\left|Q_{1}\right|=q$. Thus $\mathfrak{A}_{P_{1} Q_{1} / Q_{1}} \cong \mathbb{Z}\left[C_{p}\right]<\mathbb{Z}\left[C_{p}\right]$ since if $\mathfrak{A}_{P_{1} Q_{1} / Q_{1}} \cong \mathbb{Z}\left[C_{p}^{2}\right]$ we may apply Proposition 4.1 of [15] and these are the only $p$-Schur rings over $\mathbb{Z}_{p}^{2}$. Further it follows from $\left|Q_{1}\right|=q$ that $\bar{H} \cong C_{p}^{3}$.

The S-ring $\mathfrak{A}_{\bar{H}}$ is a Schurian $p$-S-ring over the group $\bar{H} \cong C_{p}^{3}$. The classification of such S-rings is well-known [12]. They are

$$
\begin{aligned}
& \mathfrak{B}_{1}=\mathbb{Z}\left[C_{p}^{3}\right], \\
& \mathfrak{B}_{2}=\mathbb{Z}\left[C_{p}^{2}\right] \curlywedge \mathbb{Z}\left[C_{p}\right], \\
& \mathfrak{B}_{3}=\left(\mathbb{Z}\left[C_{p}\right] \curlywedge \mathbb{Z}\left[C_{p}\right]\right) \otimes \mathbb{Z}\left[C_{p}\right], \\
& \mathfrak{B}_{4}=\mathbb{Z}\left[C_{p}\right] \curlywedge \mathbb{Z}\left[C_{p}^{2}\right], \\
& \mathfrak{B}_{5}=\mathbb{Z}\left[C_{p}\right] \curlywedge \mathbb{Z}\left[C_{p}\right] \curlywedge \mathbb{Z}\left[C_{p}\right], \\
& \mathfrak{B}_{6}=V\left(C_{p}^{3},\left(C_{p}^{3} \rtimes\langle\alpha\rangle\right)_{e}\right)
\end{aligned}
$$

Here $\alpha \in \operatorname{Aut}\left(C_{p}^{3}\right)$ is an automorphism of order $p$ which has $p$ fixed points. We can exclude the S-ring $\mathcal{B}_{6}$, because in this case the group $\bar{G}$ is not $\preceq \bar{H}$-minimal.

It follows from $\mathfrak{A}_{Q_{1} P_{1} / Q_{1}} \cong \mathbb{Z}\left[C_{p}\right] \curlywedge \mathbb{Z}\left[C_{p}\right]$ that there exists an $\overline{\mathfrak{A}}$-subgroup of order $p^{2}$ on which the induced Schur ring is isomorphic to $\mathbb{Z}\left[C_{p}\right]<\mathbb{Z}\left[C_{p}\right]$. This excludes $\overline{\mathfrak{A}} \cong \mathfrak{B}_{1}$ or $\mathfrak{B}_{2}$.

It remains to settle the cases $\overline{\mathfrak{A}} \cong \mathfrak{B}_{i}, i=3,4,5$.

The inclusion $\operatorname{Aut}_{\bar{H}}(\overline{\mathfrak{A}})^{\overline{P_{1}}} \leqslant \operatorname{Aut}_{\overline{P_{1}}}\left(\mathfrak{A}_{\overline{P_{1}}}\right)$ is trivial. To prove the inverse inclusion we note that each of the $S$-rings $\mathfrak{B}_{i}, i=3,4,5$ is cyclotomic. In particular this implies that $\operatorname{Aut}_{\bar{H}}(\overline{\mathfrak{A}})$ acts transitively on each basic set of $\overline{\mathfrak{A}}$. Therefore $\operatorname{Aut}_{\bar{H}}(\overline{\mathfrak{A}})^{F}$ is nontrivial whenever the induced S-ring $\overline{\mathfrak{A}}_{F}$ is non-trivial for any $\overline{\mathfrak{A}}$-subgroup $F$. This implies that $\operatorname{Aut}_{\bar{H}}(\overline{\mathfrak{A}})^{\overline{P_{1}}}$ is non-trivial. Therefore, $p \leqslant\left|\operatorname{Aut}_{\bar{H}}(\overline{\mathfrak{A}})^{\overline{P_{1}}}\right| \leqslant\left|\operatorname{Aut}_{\overline{P_{1}}}\left(\mathfrak{A}_{\overline{P_{1}}}\right)\right|$.

On the other hand, Aut $\overline{P_{1}}\left(\mathfrak{A}_{\overline{P_{1}}}\right)=\operatorname{Aut}_{C_{p}^{2}}\left(\mathbb{Z}\left[C_{p}\right]<\mathbb{Z}\left[C_{p}\right]\right)$ is contained in a Sylow $p$-subgroup of $\operatorname{Aut}\left(C_{p}^{2}\right) \cong G L_{2}(p)$. Since the latter one has order $p$, we conclude that $\left|\operatorname{Aut}_{\overline{P_{1}}}\left(\mathfrak{A}_{\overline{P_{1}}}\right)\right| \leqslant p$ implying $\operatorname{Aut}_{\bar{H}}(\overline{\mathfrak{A}})^{\overline{P_{1}}}=\operatorname{Aut}_{\overline{P_{1}}}\left(\mathfrak{A}_{\overline{P_{1}}}\right)$.

Therefore $\operatorname{Aut}_{\bar{H}}(\overline{\mathfrak{A}})^{\overline{P_{1}}}=\operatorname{Aut}_{\overline{P_{1}}}\left(\mathfrak{A}_{\overline{P_{1}}}\right)$ and by Theorem 2.9 of [15] the corresponding S-ring is CI. 
4.2. Proof of Theorem 4.1 In the CASE of $P_{1} Q_{1}=H$. Note, first, that $\left|H / P_{1}\right|$ is divisible by $q$.

If $\left|H / P_{1}\right| \neq q$, then by Proposition 3.4 we have $\mathfrak{A}=\mathfrak{A}_{P_{1}} \star \mathfrak{A}_{Q_{1}}$. Since both $P_{1}$ and $Q_{1} /\left(P_{1} \cap Q_{1}\right)$ are $\mathcal{E}$-groups with at most three prime factors, they are $\mathrm{CI}^{(2)}$-groups by [12] and [14]. Therefore, $\mathfrak{A}_{P_{1}}$ and $\mathfrak{A}_{Q_{1} /\left(P_{1} \cap Q_{1}\right)}$ are CI-S-rings. By Theorem $2.8 \mathfrak{A}$ is a CI-S-ring.

Assume now that $\left|H / P_{1}\right|=q$. Since $G$ is $\preceq_{H}$-minimal, its quotient $G^{H / P_{1}}$ is $\preceq_{H / P_{1}}$ minimal too. Therefore $G^{H / P_{1}} \cong C_{q}$ and $\mathfrak{A}_{H / P_{1}} \cong \mathbb{Z}\left[C_{q}\right]$. By Proposition $3.5 \mathfrak{A}=$ $\mathfrak{A}_{P_{1}} \star \mathfrak{A}_{Q_{1}}$. As before, we conclude that $\mathfrak{A}$ is a CI-S-ring.

Although the case of $q=2$ is not considered in the paper, the main result remains true also in this case.

Acknowledgements. The authors are very thankful to the referees who read the manuscript carefully. Their reports helped enormously to improve the paper.

"Application Domain Specific Highly Reliable IT Solutions" project has been implemented with the support provided from the National Research, Development and Innovation Fund of Hungary, financed under the Thematic Excellence Programme TKP2020-NKA-06 (National Challenges Subprogramme) funding scheme.

\section{REFERENCES}

[1] András Ádám, Research problem 2-10, Journal of Combinatorial Theory 2 (1967), no. 3, 393.

[2] Brian Alspach and Torrence D. Parsons, Isomorphism of circulant graphs and digraphs, Discrete Math. 25 (1979), no. 2, 97-108.

[3] László Babai, Isomorphism problem for a class of point-symmetric structures, Acta Math. Acad. Sci. Hungar. 29 (1977), no. 3-4, 329-336.

[4] László Babai and Péter Frankl, Isomorphisms of Cayley graphs. I, Combinatorics (Proc. Fifth Hungarian Colloq., Keszthely, 1976), Vol. I (Amsterdam-New York), Colloq. Math. Soc. János Bolyai, vol. 18, North-Holland, 1978, pp. 35-52.

[5] Dragomir Ž. Djoković, Isomorphism problem for a special class of graphs, Acta Math. Acad. Sci. Hungar. 21 (1970), no. 3-4, 267-270.

[6] Edward Dobson, The isomorphism problem for Cayley ternary relational structures for some abelian groups of order 8p, Discrete Math. 310 (2010), no. 21, 2895-2909.

[7] Ted Dobson, Some new groups which are not CI-groups with respect to graphs, Electron. J. Combin. 25 (2018), no. 1, Paper no. 1.12 (7 pages).

[8] Bernard Elspas and James Turner, Graphs with circulant adjacency matrices, J. Combinatorial Theory 9 (1970), 297-307.

[9] Sergei A. Evdokimov and Ilya N. Ponomarenko, Schurity of S-rings over a cyclic group and generalized wreath product of permutation groups, St. Petersbg. Math. J. 24 (2013), no. 3, 431-460.

[10] Yan-Quan Feng and István Kovács, Elementary abelian groups of rank 5 are DCI-groups, J. Combin. Theory Ser. A 157 (2018), 162-204.

[11] Christopher D. Godsil, On Cayley graph isomorphisms, Ars Comb. 15 (1983), 231-246.

[12] Mitsugu Hirasaka and Mikhail Muzychuk, An elementary abelian group of rank 4 is a CI-group, J. Combin. Theory Ser. A 94 (2001), no. 2, 339-362.

[13] Mikhail H. Klin and Reinhard Pöschel, The König problem, the isomorphism problem for cyclic graphs and the method of Schur rings, in Algebraic methods in graph theory, Vol. I, II (Szeged, 1978), Colloq. Math. Soc. János Bolyai, vol. 25, North-Holland, Amsterdam-New York, 1981, pp. 405-434.

[14] István Kovács and Mikhail Muzychuk, The group $\mathbb{Z}_{p}^{2} \times \mathbb{Z}_{q}$ is a CI-group, Comm. Algebra 37 (2009), no. 10, 3500-3515.

[15] István Kovács and Grigory Ryabov, CI-property for decomposable Schur rings over an abelian group, Algebra Colloq. 26 (2019), no. 1, 147-160.

[16] Cai Heng Li, Zai Ping Lu, and PP Pálfy, Further restrictions on the structure of finite CI-groups, J. Algebraic Combin. 26 (2007), no. 2, 161-181.

[17] Joy Morris, Elementary proof that $\mathbb{Z}_{p}^{4}$ is a DCI-group, Discrete Math. 338 (2015), no. 8, 13851393. 
The Cayley isomorphism property for $\mathbb{Z}_{p}^{3} \times \mathbb{Z}_{q}$

[18] Mikhail Muzychuk, Ádám's conjecture is true in the square-free case, J. Combin. Theory Ser. A 72 (1995), no. 1, 118-134.

[19] _ On Ádám's conjecture for circulant graphs, Discrete Math. 167/168 (1997), 497-510.

[20] Reinhard Pöschel, Untersuchungen von S-Ringen, insbesondere im Gruppenring von pGruppen, Math. Nachr. 60 (1974), 1-27.

[21] Issai Schur, Zur Theorie der einfach transitiven Permutationsgruppen, vol. 1933, Preußische Akademie der Wissenschaften, Berlin, 1933 (German).

[22] Gábor Somlai, Elementary abelian p-groups of rank $2 p+3$ are not CI-groups, J. Algebraic Combin. 34 (2011), no. 3, 323-335.

[23] Helmut Wielandt, Finite permutation groups, Academic Press, 1964, reprinted 2014.

GÁbor SomLaI, Eötvös Loránd University, Departement of Algebra and Number Theory, Pázmány Péter sétány 1/c, Budapest, 1117, Hungary

E-mail : zsomlei@caesar.elte.hu

Mikhail Muzychuk, Ben Gurion University of the Negev, Israël

E-mail : muzychuk@gmail.com 